\title{
Ultra-high resolution optical coherence tomography for monitoring tear meniscus volume in dry eye after topical cyclosporine treatment
}

This article was published in the following Dove Press journal:

Clinical Ophthalmology

19 June 2012

Number of times this article has been viewed

\section{Jianhua Wang' \\ Lele Cui' \\ Meixiao Shen' \\ Victor L Perez' \\ Michael R Wang²}

'Bascom Palmer Eye Institute, University of Miami Leonard M Miller School of Medicine, ${ }^{2}$ Electrical and Computer Engineering, University of Miami, Miami, FL, USA
Correspondence: Jianhua Wang Bascom Palmer Eye Institute, University of Miami Leonard M Miller School of Medicine, 1638 NW 10th Avenue, McKnight Building - Room 202A, Miami, FL, 33136, USA

$\mathrm{Tel}+\mathrm{I} 3054825010$

Fax +I 3054825012

Email jwang3@med.miami.edu
Purpose: This study was designed to determine the ability of ultra-high-resolution optical coherence tomography (UHR-OCT) to monitor tear meniscus volume after dry eye treatment with Restasis ${ }^{\circledR}(0.05 \%$ cyclosporine $)$.

Methods: UHR-OCT was used to image the upper and lower tear menisci (height and volume) in 14 consecutive dry eye patients at baseline. The treatment group was initiated on daily Restasis and measurements were repeated at 1 and 2 months while on treatment. All measurements were performed immediately after a blink.

Results: The baseline tear meniscus volumes were $0.40 \pm 0.19 \mu \mathrm{L}$ (upper) and $0.43 \pm 0.18 \mu \mathrm{L}$ (lower). In the treatment group, tear meniscus heights showed significant increases of both upper (post-hoc test with Bonferroni correction: $P=0.003)$ and lower $(P=0.0003)$ tear menisci, after 1 month on Restasis. The tear meniscus volumes in the treatment group after 1 month of Restasis showed significant increases of both upper $(P=0.007)$ and lower $(P=0.007)$ tear menisci. At 2 months, the increase of the tear meniscus was still evident in all measured variables compared with baseline $(P<0.05)$.

Conclusion: UHR-OCT offers a promising tool for monitoring dry eye treatment. With this method, a measurable increase in tear meniscus volume was evident after 1 month of treatment with Restasis in dry eye patients.

Keywords: tear menisci, UHR-OCT, tear dynamics, dry eye, Restasis ${ }^{\circledR}$

\section{Introduction}

Adequate tear volume on the ocular surface is essential for maintaining ocular surface integrity and comfort. The majority of tear volume is in the tear menisci, including both the upper and the lower tear meniscus. ${ }^{1}$ Tear meniscus volume has been found to be significantly lower in dry eye patients. Recently, optical coherence tomography (OCT) has been utilized to quantify tear meniscus volume and to diagnose dry eye., ${ }^{2,3}$ In addition, OCT technology has been used to evaluate tear dynamics after instillation of artificial tears ${ }^{4}$ and occlusion of the punctum. ${ }^{5}$

The topical cyclosporine ophthalmic solution (Restasis ${ }^{\circledR}$; Allergan, Irvine, CA), is a US Food and Drug Administration-approved treatment for dry eye disease. ${ }^{6}$ Its mechanism reduces inflammation of the ocular surface, resulting in increased tear production. ${ }^{6}$ Increased tear production, as measured by Schirmer's test (an invasive method), has been found in dry eye patients treated with topical cyclosporine? A noninvasive method may be useful in determining the increase in tear volume after treatment and can be used in routine clinic situations for evaluating the efficacy of treatment in individual patients. Using high-resolution OCT, we have previously 
demonstrated that the use of artificial tears does not lead to sustained increases in tear volume as blinking returns tear volume to baseline within 30 minutes of artificial tear instillation. ${ }^{4,8}$ Our previous work has also demonstrated that OCT is capable of monitoring upper and lower tear menisci in both symptomatic and nonsymptomatic patients with dry eye..$^{2,9,10}$ To the best of our knowledge, tear meniscus volume after Restasis treatment has not been examined using ultrahigh-resolution OCT (UHR-OCT). This study was designed to determine the tear meniscus volume after dry eye treatment with Restasis $(0.05 \%)$ using the novel UHR-OCT.

\section{Subjects and methods}

This prospective study was approved by the research boards of the University of Miami. Fourteen consecutive, clinically diagnosed aqueous tear deficiency (ATD) dry eye patients (seven women and seven men; mean age \pm standard deviation: $45.7 \pm 21.1$ years) were recruited for the Restasis treatment group. Key inclusion criteria included dry eye symptoms and signs (Schirmer's test: $\leq 5 \mathrm{~mm}$, staining $\geq 1$, or tear break-up time [TBUT] $\leq 10$ seconds). The Schirmer's test with anesthesia was performed. A test strip was placed inside the conjunctival sac and the patient closed their eyes for 5 minutes. The length of moisture on the test strip was measured in millimeters. After the instillation of fluorescein, TBUT was recorded through a standard slit-lamp using cobalt blue light and a yellow filter. The time for dry spots to appear after blinking was recorded. Three tests were averaged. Staining grading was done through a standard slit-lamp when TBUT was evaluated. The Center for Contact Lens Research Unit grading scale was used for ranking the scores. Patients who were treated with Restasis were excluded. Patients in the treatment group were treated with Restasis twice a day in both eyes. No additional artificial tears were used in either group.

An ultra-high-resolution $(\sim 3 \mu \mathrm{m})$ spectral domain UHROCT has been described elsewhere. ${ }^{11,12}$ A three-module, superluminescent diode light source (Broadlighter T-840-HP; Superlum, Carrigtwohill, Ireland) with a center wavelength of $840 \mathrm{~nm}$ and a full width at half maximum bandwidth of $100 \mathrm{~nm}$ was used. The sample light was delivered to a light delivery system with a telecentric design, which consisted of an X-Y galvanometer scanner (Model 6215 Moving Magnet Closed Loop Galvanometer; Cambridge Technology, Lexington, MA). The scanner was mounted with a standard slit-lamp microscope. The power of the incident light delivered into the anterior segment was lowered to $750 \mu \mathrm{W}$ to ensure the safety of the eye. The scan width was set to $12 \mathrm{~mm}$, with a depth of $3 \mathrm{~mm}$. The scan speed of the system was set to 24,000 A-scans per second. An external fixation target was used and a video system was applied to align the eye for imaging. The upper and lower tear menisci (height, cross-sectional area, volume) were imaged at the vertical meridian across the apex. After the eye was imaged at baseline, all patients were initiated on twice-daily Restasis treatment. OCT measurements were repeated at 1 and 2 months while on treatment. The patient was instructed not to instill Restasis on the study day, and all images were taken immediately after a blink.

The lab settings of the exam room were similar to previous studies. ${ }^{1,4}$ Briefly, the temperature $\left(15^{\circ} \mathrm{C}-25^{\circ} \mathrm{C}\right)$ and humidity $(30 \%-50 \%)$ in a small consulting room were controlled by central air conditioning and two humidifiers. Ambient room light was used while imaging each subject. The images (Figure 1) showing both upper and lower tear menisci were processed with custom software, to yield upper and lower tear menisci heights and cross-sectional areas. Tear volumes in upper and lower tear menisci were calculated as described in previous studies. ${ }^{1,2}$ The volume was calculated as the product of meniscus cross-sectional area and eyelid length, obtained from a digital photograph of the eye taken during the baseline visit. As upper and lower lids are also curved in the third dimension, a multiplication factor of 1.294 was used, as suggested by Tiffany et al. ${ }^{13}$

Data analysis was conducted using Statistica software (v. 10; StatSoft, Tulsa, OK). Two-way repeated-measures analysis of variance was used for overall effects and post-hoc paired $t$-tests, with Bonferroni correction were used to determine whether there were pair-wise differences $(P<0.05)$ after the dry eye treatment.

\section{Results}

In the treatment group (Table 1 and Figure 2), tear meniscus heights showed significant increases of both upper (posthoc test with Bonferroni correction: $P=0.003$ ) and lower $(P=0.0003)$ tear menisci after 1 month on Restasis. The tear meniscus volumes in the treatment group after 1 month of Restasis showed significant increases of both upper $(P=0.007)$ and lower $(P=0.007)$ tear menisci. At 2 months, the increase of the tear meniscus was still evident in all measured variables compared with baseline $(P<0.05)$ (Figures 3 and 4$)$. The baseline tear meniscus volumes were $0.40 \pm 0.19 \mu \mathrm{L}$ (upper) and $0.43 \pm 0.18 \mu \mathrm{L}$ (lower) (Figure 4 ). At 2 months, the upper and lower tear meniscus volumes were $0.56 \pm 0.26 \mu \mathrm{L}$ and $0.69 \mu \mathrm{L} \pm 0.37 \mu \mathrm{L}$, respectively (Figure 4). 


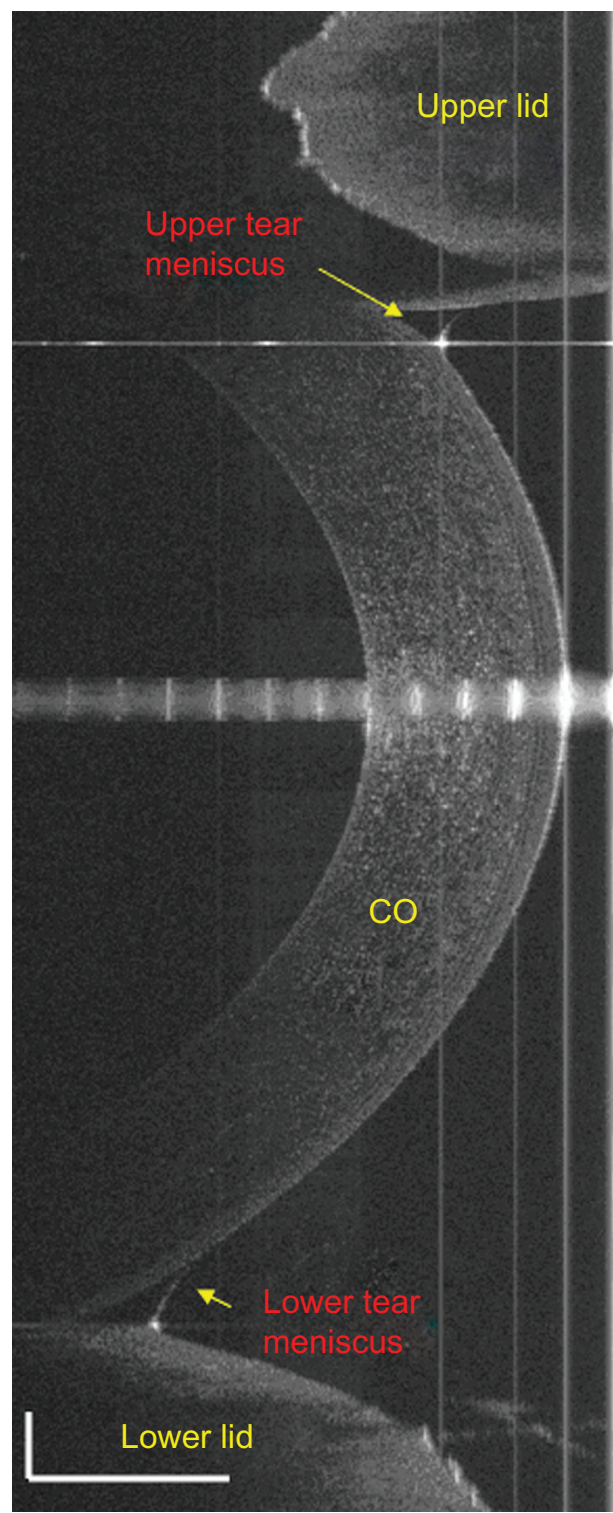

Figure I Upper and lower tear menisci, imaged with ultra-high-resolution optica coherence tomography.

Notes: A custom-built $3 \mu \mathrm{m}$ resolution ultra-high-resolution optical coherence tomography was used to image both upper and lower tear menisci. The boundaries of the tear meniscus were clearly visualized. The bars represent a $500 \mu \mathrm{m}$ scale. Abbreviation: $\mathrm{CO}$, cornea.

\section{Discussion}

Many clinical tests have been used to evaluate the tear system and, ultimately, to diagnose dry eye. ${ }^{14,15}$ These tests include tear break-up time for tear stability, ${ }^{14,15}$ Schirmer's test for tear production, ${ }^{16}$ and ocular surface staining to evaluate damage on the ocular surface. ${ }^{17}$ Conflicting results among these tests have often been observed and agreements have been proven poor. ${ }^{17,18}$ The tear system is highly dynamic and may be influenced by many internal and external factors.
Table I Upper and lower tear meniscus volumes (mean \pm standard deviation) before and after treatment with Restasis ${ }^{\circledR}$

\begin{tabular}{llll}
\hline & Baseline & I month & 2 months \\
\hline LTMV & $0.43 \pm 0.18$ & $0.62 \pm 0.28$ & $0.69 \pm 0.37$ \\
UTMV & $0.40 \pm 0.19$ & $0.59 \pm 0.40$ & $0.56 \pm 0.26$
\end{tabular}

Note: Unit of measurement, microliter $(\mu \mathrm{L})$.

Abbreviations: LTMV, lower tear meniscus volume; UTMV, upper tear meniscus volume.

Some issues in these clinical tests, such as the use of dye and reflex production of tears, may contribute to poor specificity and sensitivity. The use of fluorescein might cause reflex tearing, resulting in great variation. ${ }^{19,20}$ Local anesthesia might alter the tear system, including tear secretion and drainage, possibly resulting in conflicting results in the Schirmer's test.

Decreased tear meniscus has been previously demonstrated in dry eye patients. ${ }^{2,3,21}$ Tear meniscus measurements made by slit-lamp ${ }^{22}$ and Tearscope (Keeler Inc, Windsor, UK), especially for the lower tear meniscus, have been used to evaluate the tear system. ${ }^{23}$ OCT is a noninvasive and noncontact imaging modality that has been used for imaging the tear film and tear meniscus. ${ }^{4}$ Using a custom-built, real-time OCT instrument with a wide scan width, Wang and associates were the first to image both upper and lower tear menisci simultaneously ${ }^{4}$ and to investigate tear dynamics during the blink cycle. ${ }^{1}$ OCT has also been used to quantify tear dynamics and tear meniscus for the diagnosis of dry eye ${ }^{2,3}$ and for the evaluation of tear dynamics after instillation of artificial tears ${ }^{4}$ or occlusion of the punctum. ${ }^{5}$ With the use of high-resolution OCT, we have shown that tear meniscus curvature and height show good diagnostic accuracy, with more than $87 \%$ for both specificity and sensitivity in the diagnosis of dry eye. ${ }^{2}$ Due to its noninvasive and noncontact nature, OCT appears to be very suitable for evaluating tear meniscus volume without any stimulus for possible reflexive tearing.

In the present study, we utilized a high-resolution OCT for the first time to evaluate changes in the tear meniscus following the use of a topical cyclosporine in dry eye patients. A measurable increase of tear meniscus volume was found after 2 months of Restasis treatment. This is consistent with prior studies, which have demonstrated an increase of $10 \mathrm{~mm}$ or more in Schirmer's test in $15 \%$ of patients versus $5 \%$ in control patients. ${ }^{6,7}$ Treatment with topical cyclosporine has been found to reduce the expression of proinflammatory cytokines, cell surface markers of $\mathrm{T}$ lymphocytes, and apoptotic cells in dry eye patients. ${ }^{6}$ Improvement of dry eye symptoms and signs has also been demonstrated. ${ }^{7}$ 

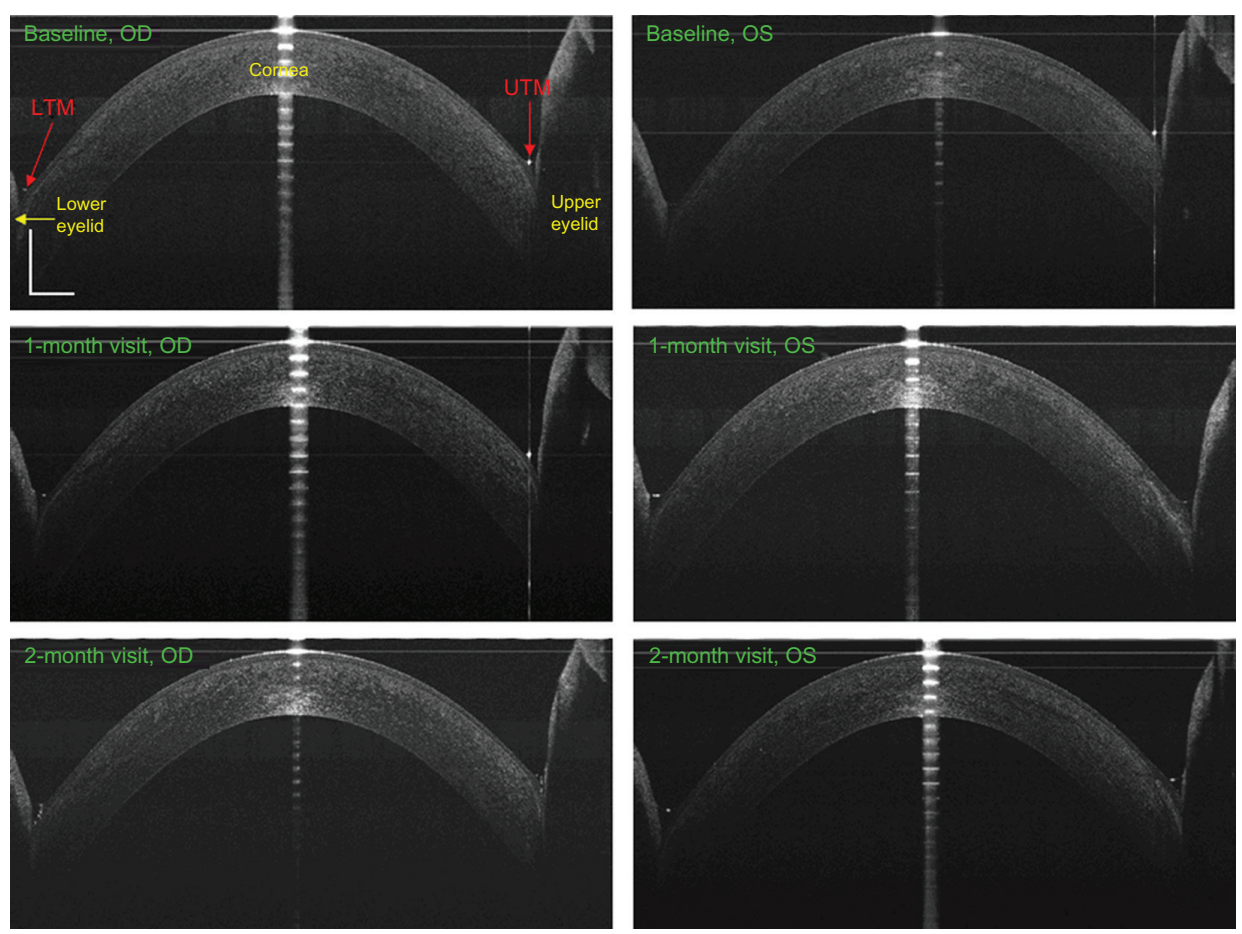

Figure 2 Tear menisci before and after 2 months of Restasis ${ }^{\circledR}$ treatment.

Notes: The upper (UTM) and lower (LTM) tear menisci were imaged at baseline, I, and 2 months from both eyes of a dry eye subject. The tear menisci appeared increased at $\mathrm{I}$ and 2 months, compared to baseline. Bars $=500 \mu \mathrm{m}$.

Abbreviations: OD, oculus dexter; OS, oculus sinister.

In the present study, after 1 month of Restasis treatment, the upper tear meniscus volume in the treatment group $(0.59 \mu \mathrm{L}$ at 1 month and $0.56 \mu \mathrm{L}$ at 2 months) increased to a value similar to normal eyes tested in an earlier study $(0.58 \mu \mathrm{L}) .{ }^{1}$ With increased tear production, the upper tear meniscus should increase first, before the tears are transferred to the lower tear meniscus. The lower tear meniscus continued to improve at 1 and 2 months; though, at the final visit, the lower tear meniscus volume $(0.69 \mu \mathrm{L})$ had still not approached that of a normal group $(0.81 \mu \mathrm{L}) .{ }^{1}$ This suggests that 2 months of treatment alone may not achieve full recovery to a normal level. However, age may be a factor when normal volume of the tear meniscus is considered and additional studies, perhaps of longer duration, with agematched controls are necessary to fully address whether the treatment can fully restore the tear production to an age-matched normal level.

The tear menisci act as reservoirs; tears newly secreted by the lacrimal gland flow first to the upper tear meniscus and then to the lower tear meniscus via the lid junctions. ${ }^{1,24}$ During blinking, the upper and lower tear menisci are mixed and form a thin tear film on the ocular surface. ${ }^{1}$ It has been documented that artificial tears do not lead to sustained increases in tear volume, as blinking returns tear volume
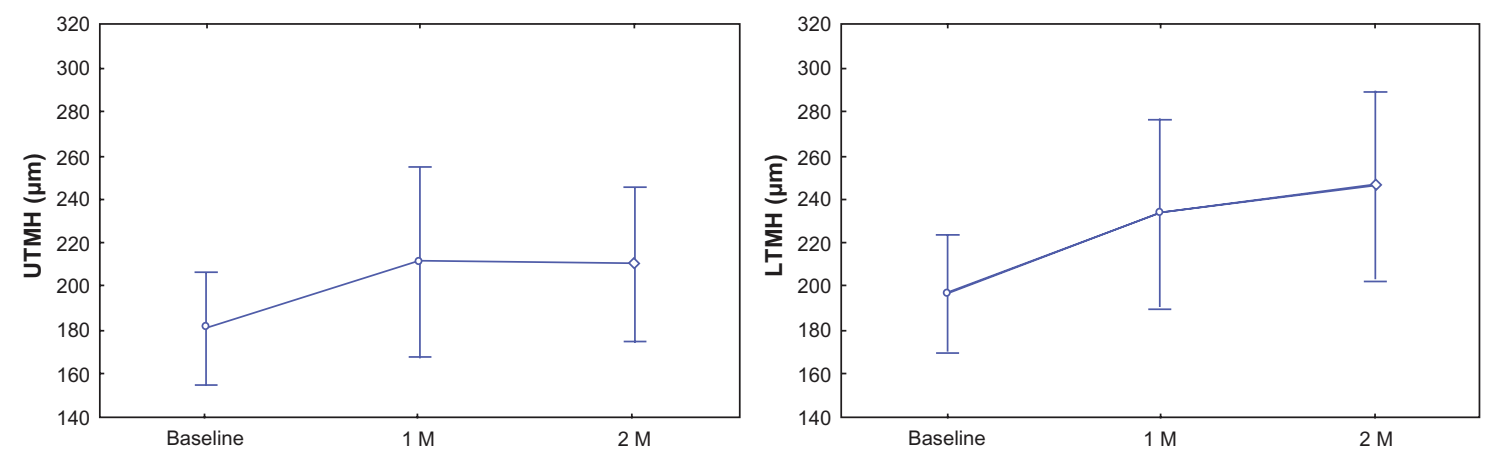

Figure 3 Upper and lower tear menisci before and after Restasis ${ }^{\circledR}$ treatment in dry eye patients.

Notes: Upper (UTMH) and lower (LTMH) tear meniscus heights were significantly increased at I and 2 months compared with baseline $(P<0.05)$. Bars represent $95 \%$ confidence intervals. 

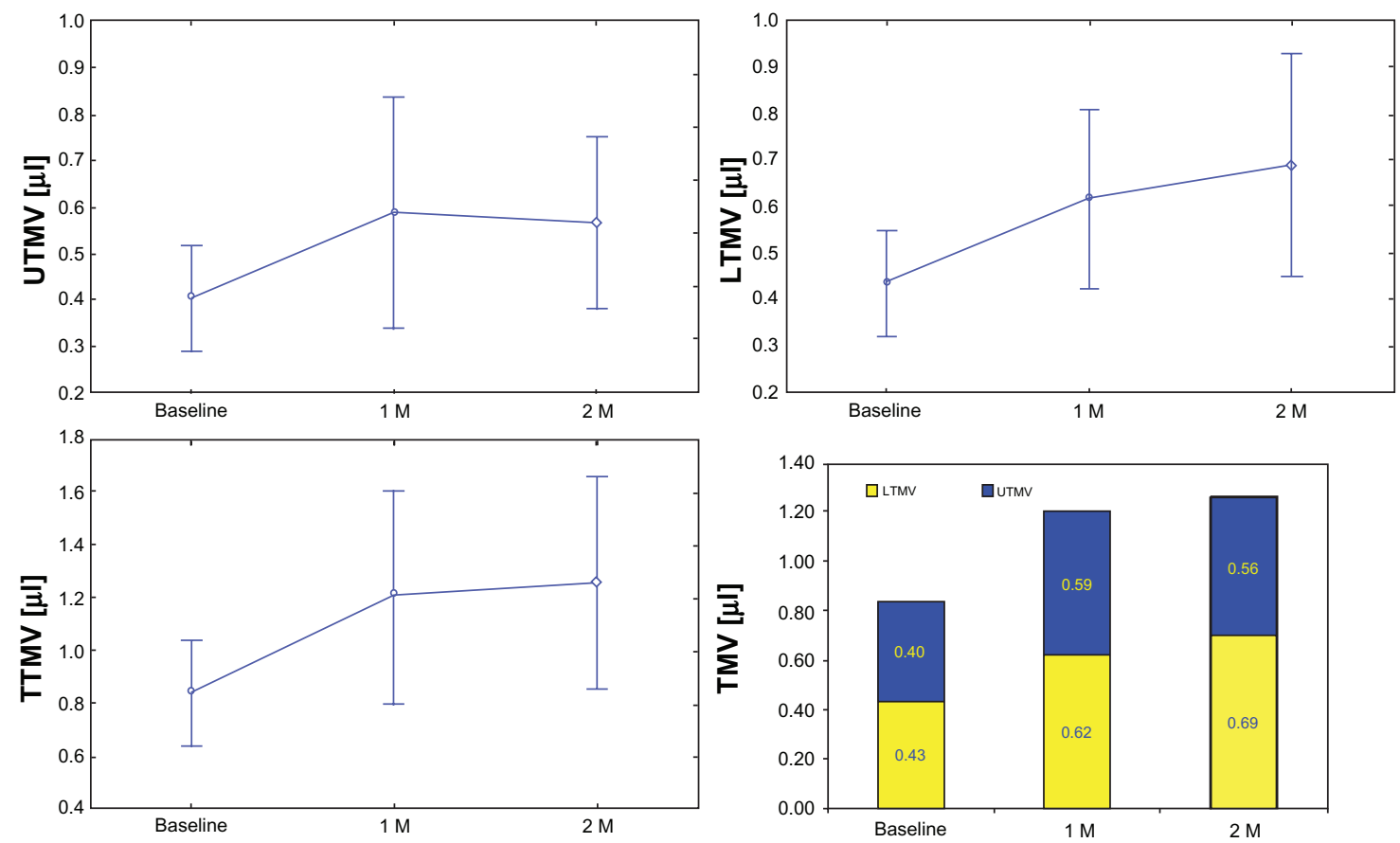

Figure 4 Tear meniscus volumes before and after Restasis ${ }^{\circledR}$ treatment in dry eye patients.

Notes: Both upper and lower tear meniscus volumes (UTMV and LTMV) and total tear meniscus volume (TTMV) were significantly increased at I and 2 months, compared to baseline $(P<0.05)$. Bars represent $95 \%$ confidence intervals.

to baseline within a very short period after artificial tear instillation. ${ }^{4,8}$ Wang and associates tracked tear meniscus volumes with four different artificial tears and found that the instilled tear volume in the tear meniscus appeared to decrease to baseline level within 30 minutes. ${ }^{8}$ In the present study, all measurements were taken at least 2 hours after drop instillation to avoid possible effects from instillation of the fluid vehicle of Restasis.

There are some limitations in the present study. Tear volume was estimated using lid length and tear meniscus area at the central lid, as we did in a previous study, ${ }^{8}$ which could have resulted in some measurement error. Significant increases of the tear meniscus after treatment, compared to baseline, were evident, even with the measurement error. Further, blinking rate is a factor of tear dynamics ${ }^{25,26}$ but is difficult to control. Normal blinking was allowed during imaging, and the image was acquired shortly after blinking, which may lower the impact on the tear meniscus of the blinking rate. Further studies will address this issue by controlling the blinking interval during imaging. Finally, this was a pilot study to determine the tear volume after dry eye treatment. In this study, the subjects were not randomly chosen and no control group was used. In addition, this was not a double-blind study. However, the study will still provide useful information, possibly leading to well-designed clinical trials on the efficacy of dry eye treatment.

\section{Conclusion}

UHR-OCT is capable of monitoring tear meniscus volume after dry eye treatment with Restasis $(0.05 \%$ cyclosporine $)$. A measurable increase in tear meniscus volume was evident after 2 months of treatment with Restasis in ATD dry eye patients.

\section{Acknowledgments}

This study was supported by research grants from Allergan Inc, the National Institutes of Health (NIH grant P30 EY014801), and Research to Prevent Blindness (New York, NY). We wish to thank Dr Britt Bromberg of Xenofile Editing (New Orleans, LA), for providing editing services for this manuscript.

\section{Disclosure}

Jianhua Wang is a recipient of the research grant from Allergan Inc. The authors have no proprietary interest in any materials or methods described within this article.

\section{References}

1. Palakuru J, Wang J, Aquavella J. Effect of blinking on tear dynamics. Invest Ophthalmol Vis Sci. 2007;48(7):3032-3037.

2. Shen M, Li J, Wang J, et al. Upper and lower tear menisci in the diagnosis of dry eye. Invest Ophthalmol Vis Sci. 2009;50(6):2722-2726.

3. Yuan Y, Wang J, Chen Q, Tao A, Shen M, Shousha MA. Reduced tear meniscus dynamics in dry eye patients with aqueous tear deficiency. $\mathrm{Am}$ J Ophthalmol. 2010;149(6):932-938. 
4. Wang J, Aquavella J, Palakuru J, Chung S. Repeated measurements of dynamic tear distribution on the ocular surface after instillation of artificial tears. Invest Ophthalmol Vis Sci. 2006;47(8):3325-3329.

5. Chen F, Shen M, Chen W, et al. Tear meniscus volume in dry eye after punctal occlusion. Invest Ophthalmol Vis Sci. 2010;51(4):1965-1969.

6. Donnenfeld E, Pflugfelder SC. Topical ophthalmic cyclosporine: pharmacology and clinical uses. Surv Ophthalmol. 2009;54(3): 321-338.

7. Sall K, Stevenson OD, Mundorf TK, Reis BL. Two multicenter, randomized studies of the efficacy and safety of cyclosporine ophthalmic emulsion in moderate to severe dry eye disease. CsA Phase 3 Study Group. Ophthalmology. 2000;107(4):631-639.

8. Wang J, Simmons P, Aquavella J, et al. Dynamic distribution of artificial tears on the ocular surface. Arch Ophthalmol. 2008;126(5):619-625.

9. Chen F, Wang J, Chen W, Shen M, Xu S, Lu F. Upper punctal occlusion versus lower punctal occlusion in dry eye. Invest Ophthalmol Vis Sci. 2010;51(11):5571-5577.

10. Chen Q, Wang J, Shen M, et al. Lower volumes of tear menisci in contact lens wearers with dry eye symptoms. Invest Ophthalmol Vis Sci. 2009;50:3159-3163.

11. Chen Q, Wang J, Tao A, Shen M, Jiao S, Lu F. Ultrahigh-resolution measurement by optical coherence tomography of dynamic tear film changes on contact lenses. Invest Ophthalmol Vis Sci. 2010;51(4): 1988-1993.

12. Wang J, Jiao S, Ruggeri M, Shousha MA, Chen Q. In situ visualization of tears on contact lens using ultra high resolution optical coherence tomography. Eye Contact Lens. 2009;35(2):44-49.

13. Tiffany JM, Todd BS, Baker MR. Computer-assisted calculation of exposed area of the human eye. Adv Exp Med Biol. 1998;438: 433-439.

14. Lemp MA, Hamill JR Jr. Factors affecting tear film breakup in normal eyes. Arch Ophthalmol. 1973;89(2):103-105.
15. Begley CG, Himebaugh N, Renner D, et al. Tear breakup dynamics: a technique for quantifying tear film instability. Optom Vis Sci. 2006; 83(1):15-21.

16. Clinch TE, Benedetto DA, Felberg NT, Laibson PR. Schirmer's test. A closer look. Arch Ophthalmol. 1983;101(9):1383-1386.

17. McCarty CA, Bansal AK, Livingston PM, Stanislavsky YL, Taylor HR. The epidemiology of dry eye in Melbourne, Australia. Ophthalmology. 1998;105(6):1114-1119.

18. Nichols KK, Nichols JJ, Zadnik K. Frequency of dry eye diagnostic test procedures used in various modes of ophthalmic practice. Cornea. 2000;19(4):477-482.

19. Mengher LS, Bron AJ, Tonge SR, Gilbert DJ. Effect of fluorescein instillation on the pre-corneal tear film stability. Curr Eye Res. 1985; 4(1):9-12.

20. Patel S, Murray D, McKenzie A, Shearer DS, McGrath B. Effects of fluorescein on tear breakup time and on tear thinning time. Am J Optom Physiol Opt. 1985;62(3):188-190.

21. Mainstone JC, Bruce AS, Golding TR. Tear meniscus measurement in the diagnosis of dry eye. Curr Eye Res. 1996;15(6):653-661.

22. Golding TR, Bruce AS, Mainstone JC. Relationship between tearmeniscus parameters and tear-film breakup. Cornea. 1997;16(6): 649-661.

23. Fodor E, Hagyó K, Resch M, Somodi D, Nemeth J. Comparison of Tearscope-plus versus slit lamp measurements of inferior tear meniscus height in normal individuals. Eur J Ophthalmol. 2010;20(5): 819-824.

24. Doane MG. Blinking and the mechanics of the lacrimal drainage system. Ophthalmology. 1981;88(8):844-851.

25. Sahlin S, Laurell CG, Chen E, Philipson B. Lacrimal drainage capacity, age and blink rate. Orbit. 1998;17(3):155-159.

26. Sahlin S, Chen E. Gravity, blink rate, and lacrimal drainage capacity. Am J Ophthalmol. 1997;124(6):758-765.
Clinical Ophthalmology

\section{Publish your work in this journal}

Clinical Ophthalmology is an international, peer-reviewed journal covering all subspecialties within ophthalmology. Key topics include: Optometry; Visual science; Pharmacology and drug therapy in eye diseases; Basic Sciences; Primary and Secondary eye care; Patient Safety and Quality of Care Improvements. This journal is indexed on

\section{Dovepress}

PubMed Central and CAS, and is the official journal of The Society of Clinical Ophthalmology (SCO). The manuscript management system is completely online and includes a very quick and fair peer-review system, which is all easy to use. Visit http://www.dovepress.com/ testimonials.php to read real quotes from published authors. 